\title{
A holistic approach to intelligent automated control
}

\author{
I. Alarcon, P. Gomez, M. Campos, J.A. Aguilar, S. Romero and P.
} Serrahima

Instituto de Ingenieria del Conocimiento. Mod. C-XVI, p.4, UAM, Cantoblanco, E-28049 Madrid (Spain). Tel: +34 1397 3973. Fax: +3413973972.

e-mail: idoia@helena.iic.uam.es

P. T. Breuer

Departamento de Ingenieria de Sistemas Telematicos, E.T.S.I.T., Universidad Politecnica de Madrid, Ciudad Universitaria, E-28040 Madrid (Spain)

e-mail:ptb@dit.upm.es

\begin{abstract}
Our belief is that industrial problems require holistic solutions if AI based control is to become an everyday reality. We have designed and implemented a software architecture which supports heterogenous AI subsystems and which is now controlling a working plant. The support architecture is associated with (1) a design methodology and (2) an underlying conceptual model of an integrated system. In the latter, a reactive system is seen as consisting of layers consisting of interacting elements called 'basic control tasks'. Designing a control system to this model requires the analyst to consider the plant, its environment and it components as a whole.
\end{abstract}

Keywords

Integration, Artificial Intelligence, industrial control.

\section{INTRODUCTION}

Better automation might be the key to the improvement of industrial competitiveness, but, in most industrial plants, full automation is not considered possible because intelligent decisions in a larger setting play an essential part in the managerial and control process. 
The favoured approach is to rely on human overseers for intelligent input that can take account of more broadly-based considerations than may be programmed into conventional automated control designs. Although some instances of installations with AI based control subsystems do exist (Dubas, 1990), (Larsen, 1980), (Sofge and White, 1990), our belief is that industrial control problems require holistic solutions. They should involve the cooperative application of at least several AI techniques to be successful, and different techniques are appropriate in different parts of the management and control process. The problem of optimizing the behaviour of an industrial plant and its environment as a combined entity is inherently complex and multi-faceted. Seen from this viewpoint, the lack of a common framework and a software architecture for the support of AI-based industrial control designs must have contributed to their lack of penetration in the industrial arena.

This paper describes a framework that has being developed and is being used at the Knowledge Engineering Institute (IIC) for the support of heterogeneous intelligent systems applied to the management and control of whole industrial installations. Section 2 describes the underlying conceptual model. Section 3 describes the basic repeating unit that comprises instances of the model and Section 4 sets out a methodology for design under the framework. Section 5 gives some details of the software used to provide the supporting architecture for the installed systems.

\section{CONCEPTUAL FRAMEWORK}

The approach presented in this paper is founded on the framework provided by a general conceptual model of intelligent control (Alarcon et al,, 1993). The emphasis here is on the optimization of production taking into account a holistic measure of plant performance (including, for example, considerations of environmental safety, maintainability, etc.).

The framework formalizes the model. It provides a language in which the tasks performed by the control and production team of an industrial plant may be described. Like the underlying model, it is grounded in real daily problems and situations, and hooks for the actions required to solve or deal with them are part of the framework.

The conceptual model represents a problem oriented approach. Problems, together with their dynamics and related control actions are at the heart of the model. Problems are associated in the model with a set of activities and control actions. The latter have to be performed in a specific order and under tightly determined conditions in order to solve the problem. Here, problem has a general sense. It means any situation that can be modified (normally enhanced) by the actions performed by the plant staff. Some examples are: detection of failures in the process, improvement of the quality of the final product, treatment of the environmental impact, etc.

(In the approach proposed here, an entire group of such problem-oriented activities is analyzed and represented during the design of an intelligent control system.)

Our approach assumes that one isolated technique cannot solve the entire control problem and that more than one technique must be applied depending on the type of problem and the 
nature of the data/knowledge/information available. Because it is presently lacking, we have proposed a conceptual framework that facilitates cooperation among techniques in a natural way.

We have taken into account a particular aspect of process and management control in devising the model: namely, the hierarchical organization of the human staff and their industrial role. The conceptual model and the framework it supports are divided into three layers wherein all problems and tasks find a niche (see figure 1). These layers are, in order of lowest to highest:

\section{Operational layer}

This covers all reactive tasks, i.e. those problems or situations normally carried out by one single operator that require a quick action. A task in this layer usually concerns a single piece of equipment, a single input and a single output.

\section{Tactical Layer}

This covers more complex problems that need to be followed up until successful resolved and more than one control action might have to be carried out. Generally, however, the events treated at this level are local in extent, i.e. they affect a specific subsystem. A subsystem

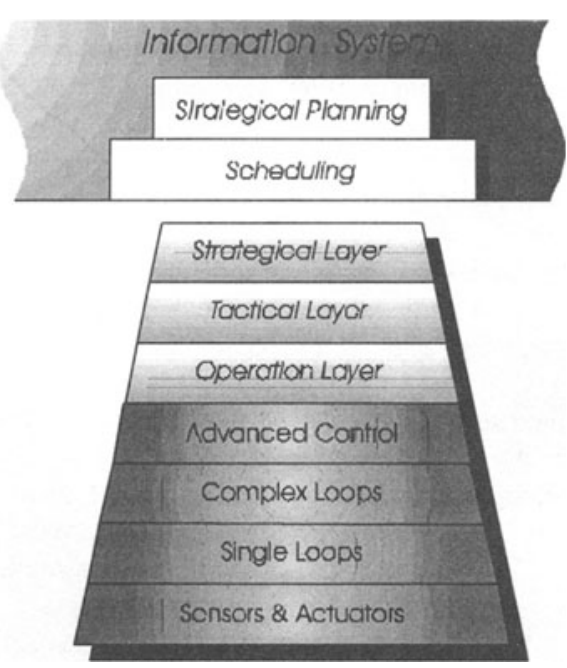

Figure 1 Control hierarchy. is defined as the set of one or more pieces of equipment and associated transfer lines. A piece of equipment is a basic unit of the plant. Given a specific problem or situation, a tactic is defined as a set of control actions that can be performed sequentially or simultaneously and which are aimed at resolving the situation. The intelligent system dynamically creates a tactic tailored to the problem and the current situation and starts recommending or performing it.

The actions that compose the tactic will be those that are defined as possible to solve this particular problem and which are not being performed or already recommended. After a certain time - needed to reconnoitre the effect of the action - it is decided whether to change the tactic or to continue with it. Account is taken of the evolving situation and the alternative sequences of possibly applicable actions in order to arrive at a prediction. This procedure is iterated until the problem is solved.

\section{Strategic Layer}

This layer has a global view. It informs lower levels about the overall strategy that is being followed or the one to be followed next. It also decides when it is necessary to change the 
overall strategy, taking into account the state of the plant and the global objectives. Recall that the global objectives are minimization of a chosen measure of performance subject to constraints on ancillary aspects. It may also decide when to change particular tactics aimed at particular subsystems.

The responsibility of this layer is to optimize the whole production task, in particular those objectives related to production, maintenance, quality, efficiency and safety. The categories of measured quantities that are considered by this layer are detailed below:

- production quantity;

- quality (this category covers both internal and external criteria of quality and, in particular, external constraints related to contracts and sales);

- efficiency (structural and staff costs are not considered, but process costs such as energy and materials are);

- maintenance costs (including evaluations of ongoing wear and predicted downtime);

- safety considerations.

The latter includes all safety criteria affecting equipment, the whole installation, the plant staff, and third parties that might be affected. All aspects related to the pollution and the environment in general are included here.

These five categories are considered to form a network of constrained variables, as seen in figure 2. All or most of the constraints relate only variables in different categories. In optimization, the focus is on one or two of them only, but all the constraints must be considered. The network of constraints is solved by means of a rule based system and

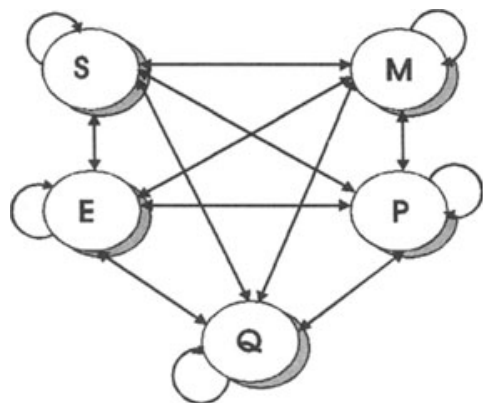

Figure 2 Plant global objectives constrained optimization techniques.

Also, because global strategic objectives may be inherently difficult to define, express or measure accurately, a computationally tractable representation does duty for them. Each objective is represented by means of a so-called logistic table. The table assigns quantities (which may be discrete classifications such as 'good', or 'warm') to the variables which comprise the measure of the objective. For example, the elements of the logistic table related to production are: assessments of availability of different products and materials, hard statistics, financial measures and production rates, and external, physical and time constraints.

A logistic table is built for each one of the strategic objectives considered. These tables are related in the sense that the same underlying variable may be reflected in more than one table. Entries in the table may be constructed by fuzzy reasoning from contributing quantities or qualities. Note that it is always the case that each overall strategic objective will be represented in the table. Both current values and boundary value (the limit that must never be surpassed) appear. 
A fuzzy value is assigned to the level of fulfillment of the strategic objectives and a rule-based method reasons with these fuzzy values in order to suggest a possible action.

\section{BASIC ELEMENTS IN THE CONCEPTUAL MODEL}

Each layer of the conceptual model is constructed from the same generic classes of object, specialized and organized in different ways. The objects are called basic control tasks.

A basic control task is oriented towards solving a unique goal with a particular set of activities and persists in a specific layer of the process control hierarchy for a specific time.

The basic control task has a consistent structure through each of the layers in the control hierarchy. The concept lends a problem oriented flavour to our approach - allowing ourselves to give to the word 'problem' a broader meaning than normal.

Another important aspect of the concept is that it serves, in the software architecture built upon the conceptual model, as the vehicle of cooperation among differing technologies. A basic control task is composed of many (so-called) slots. Each of them represents a stage in the evolution of a problem and a place where one or more technologies can make their contributions to its solution, perhaps using results derived earlier in the lifetime of the task.

Basic control tasks constitute a common paradigm for knowledge acquisition, conceptualization, design and actuation. The same conceptual tools thus cover all these phases and this can facilitate development of the application.

\section{DESIGN METHOD}

Based on the conceptual framework described above, a methodology has been defined. It aims at providing the guidelines and support necessary for the design of applications to solve a large range of process control problems by means of the integration of heterogeneous AI techniques (Alarcon et al., 1994).

The general procedure advised by the methodology is a vertical approach. It starts at the top of the hierarchy described in the previous section and proceeds downwards through (1) an analysis and (2) a design sketch for the different layers. Then the procedure is to (3) make a pass bottom-up, defining in detail the tasks and data objects of the operational, tactical and strategic layers. A layer can start to be designed in detail once the underlying layer has been analyzed. In this way, the proper functioning of a layer can be seen to be independent of higher layers.

The first step in the analysis of the process is top-down knowledge acquisition. Identification and characterization of the problems to be tackled is carried out here. Knowledge acquisition starts with a general idea of the global objectives to be included in the strategic layer. The recommended acquisition technique for this step is the open and structured interview. Once the strategic goals of the industrial installation and their priority are known in outline, the 
situations and activities corresponding to the tactical layer are studied to better understand the process and its problems. Then, the problems related to the operational layer are identified and analyzed.

The second step is the sketch of the intelligent layers, that is the identification of local control cycles according to the dynamics of the process, the nature of the control tasks and the response time. The third phase, the core of the procedure, is carried out for each layer following a bottom-up approach, and consists of the following main steps:

\section{Exhaustive knowledge acquisition and identification of problems.}

The goal is the definition of the problems to be handled by the intelligent system that will by constituted by the basic control task objects. Amongst the generally applicable acquisition techniques (Hart, 1992), the ones favoured by our methodology are interviews, questionnaires, thinking-aloud and critical incident elucidation.

\section{Assignment of identified problems to the correct layer of the model.}

This takes into account the level of complexity of the related tasks, actors involved and required response time.

\section{Definition of simple data objects.}

The parameters and both measured and calculated variables are defined.

\section{Definition and design of basic control task objects}

Our methodology sets out several procedural guidelines with the aim of achieving the following goals in sequence:

- Definition of the basic control task's objective according to the situation or problem to be solved.

- Identification of the basic control task's type. We advise classifying the basic control tasks into one of four predefined types: preventive, corrective, optimizing and modal.

- Identification of the basic control task's class. A list of the most common tasks in process control and manufacturing is predefined and available on a per control layer and basic control task type basis, although new classes may be created by the knowledge engineer carrying through this method.

- Definition of the basic control task's slots. Once the basic control task has been defined and its layer, type and class are known, the set of its slots are defined on a per control layer and type basis. This step also defines the start and end condition for the task.

- Identification of which AI techniques are needed by the basic control task in question. Within each layer and basic control task type, for each one of the established classes, the technique that is appropriate to each slot is predefined. If more than one technique may be applied, the selection criteria depends on the type of knowledge/data/information available. The techniques generally considered are rule based systems, neural networks, fuzzy logic, model based reasoning and genetic algorithms.

Once the basic control tasks are defined and designed for the three layers, the identification of interactions among basic control tasks has to be investigated. Following upon this analysis, new basic control tasks may be created. The basic control tasks to be defined at this stage 
include (a) those modifying the status of basic control tasks allocated in lower layers; (b) those constituting a basic control task's previously defined slot and (c) basic control tasks generating a fire condition of other basic control task.

The next step is the implementation of the system.

\section{IMPLEMENTATION}

A software architecture has been implemented that supports the concepts presented in previous sections. It allows the interaction and cooperation of several heterogeneous techniques in order to solve industrial control problems (de Pablo et al., 1992).

This architecture follows the blackboard paradigm (Engelmore et al., 1988), (Jagannathan et al., 1989), (Carver and Lesser, 1992), in which a centralized and active data structure is the only means of communication among modules (knowledge sources). It serves as the vehicle for cooperative problem solving, and is responsible for data coherence inside the whole system. This architecture presents a control strategy that, without losing flexibility at the design phase, is efficient.

The blackboard contains objects representing the domain information that knowledge sources will share. It includes, for example, the status of the plant, results from numerical models of parts of the plant, predictions, diagnosis of problems, etc. The blackboard may also be coerced into forcing synchronizations in order to preserve coherence. The 'basic control task' object has been integrated into the blackboard mechanism by using the dependencies expressed within the detailed definitions of its slots to generate update and data flow instructions.

Knowledge sources ultimately update and use the information in the blackboard. In particular, knowledge sources are responsible for helping basic control tasks progress by contributing information ad services to their active phases.

\section{CONCLUSIONS}

The approach presented in this paper has been succesfully carried through in a full-scale trial at a refinery in Cartagena (Spain). Different kinds of AI modules were developed for the different kinds of problems encountered, as shown in the architecture presented in figure 3. In particular, (a) neural networks, which are able to carry out the tasks of sensor validation, forecasting, and subsystem modelling, being able to give an alternative value when the sensor credibility is very low; (b) fuzzy logic, focussing on the sensor validation and signal filtering activities; (c) model-based reasoning, dealing with diagnosis and data sensor consistency checking activities; and (d) expert systems, applied when heuristic knowledge is available and, at present, covering corrective, preventive and optimizing tasks.

Because of the generality of the approach, it is now being applied to targets within manufacturing industry with very promising results. 




Figure 3 Experimented architecture.

\section{REFERENCES}

Alarcón, I., Zaccagnini, J.L., Rodriguez, P., and de Pablo, E. (1993) A Conceptual Framework for the Integration in Intelligent Control Systems Proceedings of the IRTICS workshop, Madrid, 05/1-05/15.

Alarcón, M.I., Rodríguez, P., Almeida, L.B., Sanz, R., Fontaine, L., Gomez, P., AlamÉn, X., Nordin, P., Bejder, H. and de Pablo, E. (1994) Heterogeneous Integration Architecture for Intelligent Control Intelligent Systems Engineering Journal.

Carver, N. and Lesser, V. (1994) Evolution of Blackboard Control Architectures, Expert Systems with Applications, An International Journal, USA, Volume 7, Number 1, 1-30.

Dubas, M. (1990) Expert Sytems in Industrial Practice: Advantages and Drawbacks Expert Systems, Vol. 7, No. 3, 150-156.

Engelmore, R. and Morgan, T. (1988) Blackboard Systems Addison-Wesley, California.

Hart, A. (1992) Knowledge Acquisition for Expert Systems McGraw-Hill, Inc.

Hayes-Roth, B. (1994) Integrating Real-Time AI Techniques in Adaptative Intelligent Agents Preprints of Symposium on Artificial Intelligence in Real Time Control (AIRTC'94), Ed. A. Crespo, Valencia, Spain, October, 3-14.

Jagannathan, V., Dodhiwala, R. and Baum, L.S. (1989) Blackboard Architectures and Applications Academic Press, New York.

Larsen, P.M. (1980) Industrial Applications of Fuzzy Logic Control International Journal Man-Machine Studies, Vol. 12, 3-10.

de Pablo, E., Rodriguez, P., Alarcón, I. and Alamán X. (1992) Integrating Multiple Technologies in Real-Time Intelligent Systems ECAI'92 Workshop on Advances in Real-Time Expert System Technologies, Vienna.

Rao M. (1992) Integrated Systems for Intelligent Control Springer-Verlag, Berlin.

Sofge D. and White D. (1990) Neural Network based Process Optimization and Control Proceedings of the 29th. IEEE Conference on Decision and Control, Honolulu. 